\title{
Note on style and dates
}

All quotations from manuscripts and printed primary sources are rendered in their original spelling.

The Le Sage papers in Geneva consist essentially of notes written on the backs of playing cards and are therefore referred to by the figure and suit they correspond to.

All dates follow the 'old style' calendar as found in the original British sources, except for Continental sources, which should be understood as 'new style'. In either case, 1 January is taken to mark the beginning of the year, according to the modern practice. 